\title{
DAMA/LIBRA-phase1 results and perspectives of the phase2
}

\author{
R. Bernabei ${ }^{1,2}$, P. Belli ${ }^{1,2}$, F. Cappella ${ }^{3}$, V. Caracciolo ${ }^{3}$, R. Cerulli ${ }^{3}$, C.J. Dai ${ }^{4}$, A. d'Angelo ${ }^{5,6}$, \\ S. d'Angelo ${ }^{1,2}$, A. Di Marco ${ }^{1,2}$, H.L. He ${ }^{4}$, A. Incicchitti ${ }^{5,6}$, H.H. Kuang ${ }^{4}$, X.H. Ma ${ }^{4}$, \\ F. Montecchia ${ }^{2,7}$, X.D. Sheng ${ }^{4}$, R.G. Wang ${ }^{4}$, and Z.P. Ye ${ }^{4,8}$ \\ ${ }^{1}$ Dip. di Fisica, Università di Roma “Tor Vergata”, 00133 Rome, Italy \\ ${ }^{2}$ INFN, sez. Roma "Tor Vergata", 00133 Rome, Italy \\ ${ }^{3}$ Laboratori Nazionali del Gran Sasso, I.N.F.N., Assergi, Italy \\ ${ }^{4}$ Key Laboratory of Particle Astrophysics, Institute of High Energy Physics, Chinese Academy of \\ Sciences, PO Box 918/3, 100049 Beijing, China \\ ${ }^{5}$ Dip. di Fisica, Università di Roma "La Sapienza”, 00185 Rome, Italy \\ ${ }^{6}$ INFN, sez. Roma, 00185 Rome, Italy \\ ${ }^{7}$ Dip. di Ingegneria Civile e Ingegneria Informatica, Università di Roma “Tor Vergata”, 00133 Rome, \\ Italy \\ ${ }^{8}$ University of Jing Gangshan, Jiangxi, China
}

\begin{abstract}
The DAMA/LIBRA experiment $(\sim 250 \mathrm{~kg}$ of highly radiopure $\mathrm{NaI}(\mathrm{Tl})$ ) is running deep underground at the Gran Sasso National Laboratory (LNGS) of the I.N.F.N. Here we briefly recall the results obtained in its first phase of measurements (DAMA/LIBRA-phase1; total exposure: 1.04 ton $\times$ yr). DAMA/LIBRA-phase1 and the former DAMA/NaI (cumulative exposure: 1.33 ton $\times$ yr) give evidence at $9.3 \sigma$ C.L. for the presence of DM particles in the galactic halo by exploiting the modelindependent DM annual modulation signature. No systematic or side reaction able to mimic the exploited DM signature has been found or suggested by anyone over more than a decade. At present DAMA/LIBRAphase 2 is running with increased sensitivity.
\end{abstract}

\section{Introduction}

The DAMA project is based on the development and use of low background scintillators. In particular, the second generation DAMA/LIBRA apparatus [1-13], as the former DAMA/NaI (see for example Ref. $[8,14,15]$ and references therein), is further investigating the presence of DM particles in the galactic halo by exploiting the model independent DM annual modulation signature, originally suggested in the mid 80's [16]. At present DAMA/LIBRA is running in its phase 2 with increased sensitivity. The detailed description of the DAMA/LIBRA set-up during the phase 1 has been discussed in details in Ref. [1-4, 8].

The signature exploited by DAMA/LIBRA (the model independent DM annual modulation) is a consequence of the Earth's revolution around the Sun; in fact, the Earth should be crossed by a larger flux of DM particles around $\simeq 2$ June (when the projection of the Earth orbital velocity on the Sun velocity with respect to the Galaxy is maximum) and by a smaller one around $\simeq 2$ December (when the two velocities are opposite). This DM annual modulation signature is very effective since the effect induced by DM particles must simultaneously satisfy many requirements: the rate must contain a component modulated according to a cosine function (1) with one year period (2) and a phase peaked 


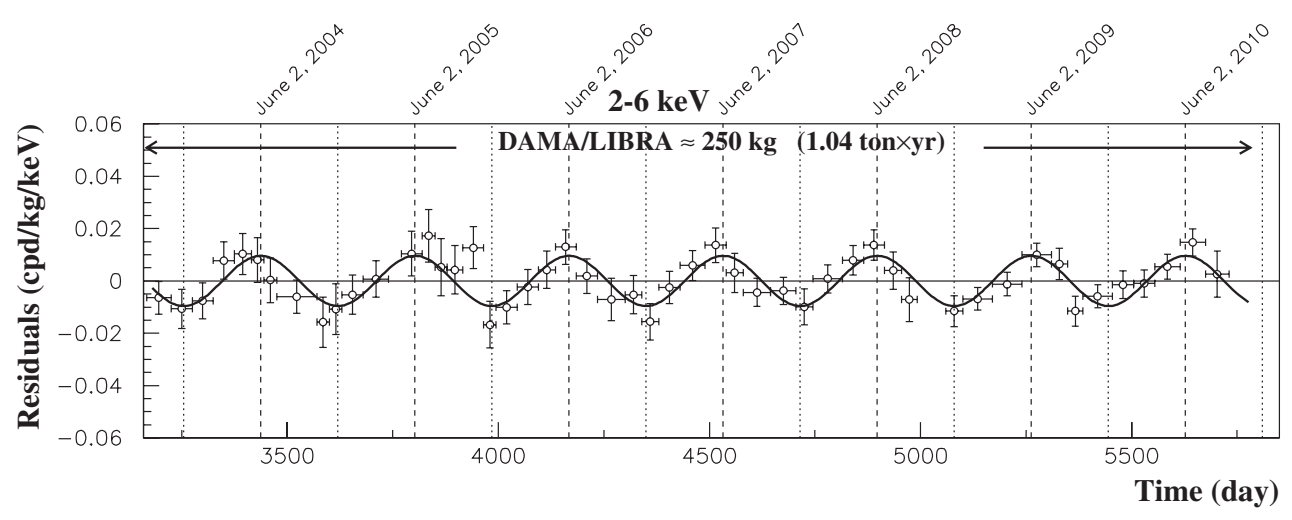

Figure 1. Experimental residual rate of the single-hit scintillation events measured by DAMA/NaI and DAMA/LIBRA-phase1 in the (2-6) keV energy interval as a function of the time. The data points present the experimental errors as vertical bars and the associated time bin width as horizontal bars. The superimposed curves are the cosinusoidal functions behaviors $A \cos \omega\left(t-t_{0}\right)$ with a period $T=\frac{2 \pi}{\omega}=$ $1 \mathrm{yr}$, a phase $t_{0}=152.5$ day (June $2^{\text {nd }}$ ) and modulation amplitudes, $A$, equal to the central values obtained by best fit on the data points. The dashed vertical lines correspond to the maximum expected for the DM signal (June $2^{\text {nd }}$ ), while the dotted vertical lines correspond to the minimum.

roughly $\simeq 2$ June (3); this modulation must only be found in a well-defined low energy range, where DM particle induced events can be present (4); it must apply only to those events in which just one detector of many actually "fires" (single-hit events), since the DM particle multi-interaction probability is negligible (5); the modulation amplitude in the region of maximal sensitivity must be $\simeq 7 \%$ for usually adopted halo distributions (6), but it can be larger (even up to $\simeq 30 \%$ ) in case of some possible scenarios such as e.g. those in Ref. $[17,18]$. Thus this signature is model independent, very discriminating and, in addition, it allows the test of a large range of cross sections and of halo densities. This DM signature might be mimicked only by systematic effects or side reactions able to account for the whole observed modulation amplitude and to simultaneously satisfy all the requirements given above. No one is available [1-4, 7, 8, 12-15, 19].

\section{The results of DAMA/LIBRA-phase1 and DAMA/Nal}

The total exposure of DAMA/LIBRA-phase 1 is 1.04 ton $\times \mathrm{yr}$ in seven annual cycles; when including also that of the first generation DAMA/NaI experiment it is 1.33 ton $\times \mathrm{yr}$, corresponding to 14 annual cycles $[2-4,8]$.

To point out the presence of the signal the time behaviour of the experimental residual rates of the single-hit scintillation events for DAMA/NaI and DAMA/LIBRA-phase1 in the (2-6) keV energy interval are plotted in Fig. 1. The $\chi^{2}$ test excludes the hypothesis of absence of modulation in the data: $\chi^{2} /$ d.o.f. $=83.1 / 50$ for the $(2-6) \mathrm{keV}$ energy interval $\left(\mathrm{P}\right.$-value $\left.=2.2 \times 10^{-3}\right)$. When fitting the single-hit residual rate of DAMA/LIBRA-phase1 together with the DAMA/NaI ones, with the function: $A \cos \omega\left(t-t_{0}\right)$, considering a period $T=\frac{2 \pi}{\omega}=1 \mathrm{yr}$ and a phase $t_{0}=152.5$ day (June $2^{\text {nd }}$ ) as expected by the DM annual modulation signature, the following modulation amplitude is obtained: $A=(0.0110 \pm$ $0.0012) \mathrm{cpd} / \mathrm{kg} / \mathrm{keV}$ corresponding to $9.2 \sigma$ C.L.. When the period, and the phase are kept free in the fitting procedure, the modulation amplitude is $(0.0112 \pm 0.0012) \mathrm{cpd} / \mathrm{kg} / \mathrm{keV}(9.3$ $\sigma$ C.L. $)$, the period $T=(0.998 \pm 0.002)$ year and the phase $t_{0}=(144 \pm 7)$ day, values well in agreement with expectations for a DM annual modulation signal. In particular, the phase is 
consistent with about June $2^{\text {nd }}$ and is fully consistent with the value independently determined by Maximum Likelihood analysis [4] $]^{1}$. The run test and the $\chi^{2}$ test on the data have shown that the modulation amplitudes singularly calculated for each annual cycle of DAMA/NaI and DAMA/LIBRA-phase1 are normally fluctuating around their best fit values [2-4].

We have also performed a power spectrum analysis of the single-hit residuals of DAMA/LIBRA-phase1 and DAMA/NaI [8], obtaining a clear principal mode in the (26) $\mathrm{keV}$ energy interval at a frequency of $2.737 \times 10^{-3} \mathrm{~d}^{-1}$, corresponding to a period of $\simeq 1$ year, while only aliasing peaks are present just above.

Absence of any other significant background modulation in the energy spectrum has been verified in energy regions not of interest for DM [4]; it is worth noting that the obtained results account of whatever kind of background and, in addition, no background process able to mimic the DM annual modulation signature (that is able to simultaneously satisfy all the peculiarities of the signature and to account for the whole measured modulation amplitude) is available (see also discussions e.g. in Ref. [1-4, 7, 8, 12, 13]).

A further relevant investigation in the DAMA/LIBRA-phase1 data has been performed by applying the same hardware and software procedures, used to acquire and to analyse the single-hit residual rate, to the multiple-hit one. In fact, since the probability that a DM particle interacts in more than one detector is negligible, a DM signal can be present just in the single-hit residual rate. Thus, the comparison of the results of the single-hit events with those of the multiple-hit ones corresponds practically to compare between them the cases of DM particles beam-on and beam-off. This procedure also allows an additional test of the background behaviour in the same energy interval where the positive effect is observed. In particular, the residual rates of the single-hit events measured over the DAMA/LIBRAphase 1 annual cycles are reported in Ref. [4], as collected in a single cycle, together with the residual rates of the multiple-hit events, in the (2-6) keV energy interval. A clear modulation satisfying all the peculiarities of the DM annual modulation signature is present in the singlehit events, while the fitted modulation amplitude for the multiple-hit residual rate is well compatible with zero: $-(0.0005 \pm 0.0004) \mathrm{cpd} / \mathrm{kg} / \mathrm{keV}$ in the same energy region (2-6) $\mathrm{keV}$. Thus, again evidence of annual modulation with the features required by the DM annual modulation signature is present in the single-hit residuals (events class to which the DM particle induced events belong), while it is absent in the multiple-hit residual rate (event class to which only background events belong). Similar results were also obtained for the last two annual cycles of the DAMA/NaI experiment [15], when the electronics allowed it. Since the same identical hardware and the same identical software procedures have been used to analyse the two classes of events, the obtained result offers an additional strong support for the presence of a DM particle component in the galactic halo.

By performing a maximum-likelihood analysis of the single-hit scintillation events, it is possible to extract from the data the modulation amplitude, $S_{m}$, as a function of the energy considering $T=1 \mathrm{yr}$ and $t_{0}=152.5$ day. Again the results have shown that positive signal is present in the (2-6) keV energy interval, while $S_{m}$ values compatible with zero are present just above; for details see Ref. [4]. Moreover, as described in Ref. [2-4, 8], the observed annual modulation effect is well distributed in all the 25 detectors, the annual cycles and the energy bins at $95 \%$ C.L. Further analyses have been performed. All of them confirm the evidence for the presence of an annual modulation in the data satisfying all the requirements for a DM signal.

\footnotetext{
${ }^{1}$ For completeness, we recall that a slight energy dependence of the phase could be expected in case of possible contributions of non-thermalized DM components to the galactic halo, such as e.g. the SagDEG stream [20-22] and the caustics [23].
} 
Sometimes naive statements were put forwards as the fact that in nature several phenomena may show some kind of periodicity. The point is whether they might mimic the annual modulation signature in DAMA/LIBRA (and former DAMA/NaI), i.e. whether they might be not only quantitatively able to account for the observed modulation amplitude but also able to contemporaneously satisfy all the requirements of the DM annual modulation signature. The same is also for side reactions. This has already been deeply investigated in Ref. [1-4] and references therein; the arguments and the quantitative conclusions, presented there, also apply to the entire DAMA/LIBRA-phase1 data. Additional arguments can be found in Ref. [7, 8, 12, 13].

No modulation has been found in any possible source of systematics or side reactions; thus, cautious upper limits on possible contributions to the DAMA/LIBRA measured modulation amplitude are summarized in Ref. [2-4]. It is worth noting that they do not quantitatively account for the measured modulation amplitudes, and also are not able to simultaneously satisfy all the many requirements of the signature. Similar analyses have also been done for the DAMA/NaI data [14, 15]. In particular, in Ref. [13] a simple and intuitive way why the neutrons, the muons and the solar neutrinos cannot give any significant contribution to the DAMA annual modulation results is outlined.

In conclusion, DAMA give model-independent evidence (at 9.3 $\sigma$ C.L. over 14 independent annual cycles) for the presence of DM particles in the galactic halo.

As regards comparisons, we recall that no direct model independent comparison is possible in the field when different target materials and/or approaches are used; the same is for the strongly model dependent indirect searches. In particular, the DAMA model independent evidence is compatible with a wide set of scenarios regarding the nature of the DM candidate and related astrophysical, nuclear and particle Physics; for examples some given scenarios and parameters are discussed e.g. in Ref. [2, 8, 14] and references therein. Further large literature is available on the topics. In conclusion, both negative results and possible positive hints are compatible with the DAMA model-independent DM annual modulation results in various scenarios considering also the existing experimental and theoretical uncertainties; the same holds for the strongly model dependent indirect approaches (see e.g. arguments in Ref. [8] and references therein).

Recently the single-hit low energy scintillation events collected by DAMA/LIBRAphase 1 have also been investigated in terms of possible diurnal effects. In particular, a diurnal effect with the sidereal time is expected for DM because of Earth rotation; this DM secondorder effect is model-independent and has several peculiar requirements as the DM annual modulation effect does. At the present level of sensitivity the presence of any significant diurnal variation and of diurnal time structures in the data can be excluded for both the cases of solar and sidereal time; in particular, the DM diurnal modulation amplitude expected, because of the Earth diurnal motion, on the basis of the DAMA DM annual modulation results is below the present sensitivity [12]. It will be possible to investigate such a diurnal effect with adequate sensitivity only when a much larger exposure will be available; moreover better sensitivities can also be achieved by lowering the software energy threshold as in the presently running DAMA/LIBRA-phase2.

For completeness we recall that other rare processes have also been searched for by DAMA/LIBRA-phase1; see for details Refs. [9-11].

\section{DAMA/LIBRA-phase2 and perspectives}

After a first upgrade of the DAMA/LIBRA set-up in September 2008, a more important upgrade has been performed at the end of 2010 when all the PMTs have been replaced with new ones having higher Quantum Efficiency (Q.E.), realized with a special dedicated development by HAMAMATSU co.. Details on the developments and on the reached performances 
in the operative conditions are reported in Ref. [6] where the feasibility to decrease the software energy threshold below $2 \mathrm{keV}$ in the new configuration has been also demonstrated.

Since the fulfillment of this upgrade, DAMA/LIBRA-phase 2 - after optimization periods - is continuously running in order: (1) to increase the experimental sensitivity lowering the software energy threshold of the experiment; (2) to improve the corollary investigation on the nature of the DM particle and related astrophysical, nuclear and particle physics arguments; (3) to investigate other signal features. This requires long and heavy full time dedicated work for reliable collection and analysis of very large exposures.

Another upgrade at the end of 2012 was successfully concluded: new-concept preamplifiers were installed, with suitable operative and electronic features. Moreover, further improvements are planned; in particular, new trigger modules have been prepared and ready to be installed.

Finally, further improvements to increase the sensitivity of the set-up can be considered; in particular, the use of high Q.E. and ultra-low background PMTs directly coupled to the $\mathrm{NaI}(\mathrm{Tl})$ crystals is an interesting possibility. This possible configuration can allow a further large improvement in the light collection and a further lowering of the software energy threshold. Moreover, efforts towards a possible highly radiopure $\mathrm{NaI}(\mathrm{Tl})$ "general purpose" experiment (DAMA/1ton) having full sensitive mass of 1 ton (we already proposed in 1996 as a general purpose set-up) have been continued in various aspects.

\section{References}

[1] R. Bernabei et al., Nucl. Instr. and Meth. A 592 (2008) 297

[2] R. Bernabei et al., Eur. Phys. J. C 56 (2008) 333

[3] R. Bernabei et al., Eur. Phys. J. C 67 (2010) 39

[4] R. Bernabei et al., Eur. Phys. J. C 73 (2013) 2648

[5] P. Belli et al., Phys. Rev. D 84 (2011) 055014

[6] R. Bernabei et al., J. of Instr. 7 (2012) P03009

[7] R. Bernabei et al., Eur. Phys. J. C 72 (2012) 2064

[8] R. Bernabei et al., Int. J. of Mod. Phys. A 28 (2013) 1330022

[9] R. Bernabei et al., Eur. Phys. J. C 62 (2009) 327

[10] R. Bernabei et al., Eur. Phys. J. C 72 (2012) 1920

[11] R. Bernabei et al., Eur. Phys. J. A 49 (2013) 64

[12] R. Bernabei et al., Eur. Phys. J. C 74 (2014) 2827

[13] R. Bernabei et al., Eur. Phys. J. C 74 (2014) 3196

[14] R. Bernabei el al., La Rivista del Nuovo Cimento 26 (1) (2003) 1-73

[15] R. Bernabei et al., Int. J. Mod. Phys. D 13 (2004) 2127

[16] K.A. Drukier et al., Phys. Rev. D 33, 3495 (1986); K. Freese et al., Phys. Rev. D 37, 3388 (1988)

[17] D. Smith and N. Weiner, Phys. Rev. D 64 (2001) 043502; D. Tucker-Smith and N. Weiner, Phys. Rev. D 72 (2005) 063509; D. P. Finkbeiner et al, Phys. Rev. D 80 (2009) 115008

[18] K. Freese et al., Phys. Rev. D 71 (2005) 043516; K. Freese et al., Phys. Rev. Lett. 92 (2004) 11301

[19] R. Bernabei et al., Eur. Phys. J. C 18 (2000) 283

[20] R. Bernabei et al., Eur. Phys. J. C 47 (2006) 263

[21] K. Freese et al., Phys. Rev. D 71 (2005) 043516; New Astr. Rev. 49 (2005) 193; astroph/0310334; astro-ph/0309279

[22] G. Gelmini, P. Gondolo, Phys. Rev. D 64 (2001) 023504

[23] F.S. Ling, P. Sikivie and S. Wick, Phys. Rev. D 70 (2004) 123503 